\title{
Pragmatic evaluation of The BMJ's visual abstracts
}

Keywords: visual abstracts, data visualisation, health, medicine, graphic design, publishing, science, evaluation, social media

The British Medical Journal has recently started making visual abstracts to summarise published research studies. These $1024 \times 1024$ px images give a quick overview of a trial's participants, design, and key findings. These visual abstracts are designed to help busy health professionals and researchers get a quick overview of newly published research. The present article describes simple pragmatic evaluations of these visual abstracts: analysis of social media stats and an opportunistic reader survey. Our goals were to identify how useful our readers found this new visual format, and whether there were any improvements we could make. The social media stats were initially very promising. Longer term performance over several visual abstracts, however, was not as strong, suggesting a possible halo effect provided by the novelty of a new presentation format. The survey proved to be a quick and valuable way of getting feedback on the design of the initial template, and resulted in several design adjustments.

\section{Background}

In 2018, The BMJ, formerly the British Medical Journal, started making visual abstracts to summarise research papers. Reader feedback has shown that quickly digestible "bite-sized" content is highly valued and the journal's infographics for educational content are popular with readers. The journal was interested in creating similar visual formats for research content to be included in articles, shared on social media, and to be used in presentations by authors and readers.

Scientific journals have been publishing visual abstracts for many years but there is only a small body of published research literature about them. The Annals of Surgery has been creating visual abstracts since July 2016 (see Figure 1). An evaluation of their social media performance found that tweets with visual abstracts had 7.7 times as many impressions and 8.4 times as many retweets, compared to tweets that included only the title of the article (Ibrahim, Lillemoe, Klingensmith, \& Dimick 2017). Marketing studies have previously found that social media posts with images are more effective than text-only posts (Cooper 2013; Pinantoan 2015), which may explain some or all of this observed effect, i.e., a different image (that is not a visual abstract) might have a similar effect.

Not all published evaluations of visual abstracts have reported positive findings. A non-randomised study of visual abstracts in the journal Molecules found that articles without visual abstracts had more PDF downloads, abstract views and total citations (Pferschy-Wenzig, 
Pferschy, Wang, Mocan, \& Atanasov 2016). The observed effect could be driven by different kinds of papers having a visual abstract, such as more early-career researchers submitting visual abstracts. The authors also suggest that more efficient "triage" by readers who have an overview from the visual abstract could lead to fewer PDFs being downloaded. The authors also note that most manuscripts had Altmetric scores of o and 1 , so the numbers involved may be small.

Cheng and colleagues found that redesigning visual abstracts published in Nano Letters enhanced readers' first impressions of a paper (Cheng, Chen, Larson, \& Rolandi 2017), suggesting that existing design practices can be improved.

\section{Methods}

Given the lack of robust evidence, but the increasing popularity in other journals, we decided to produce a limited number of visual abstracts and run some simple evaluations.

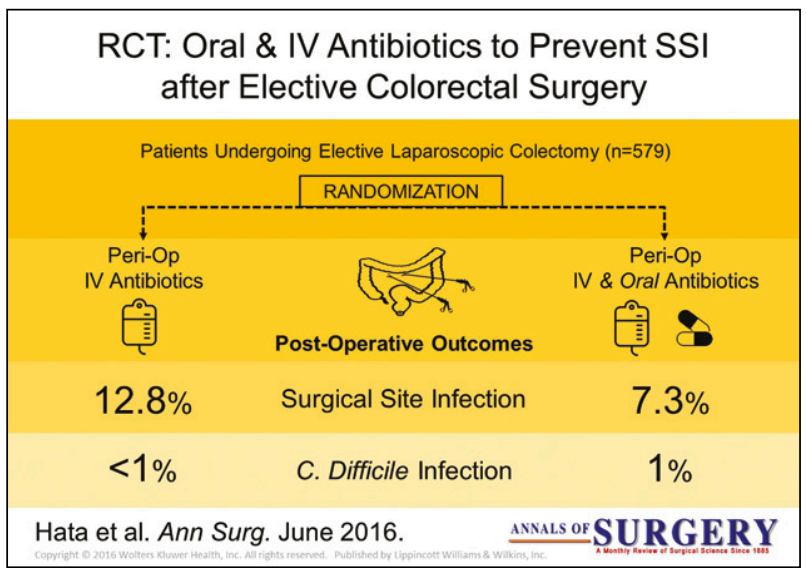

We initially chose to make visual abstracts for articles reporting randomised controlled trials only. These were selected because they are high-value study designs, often thought of as the gold standard for medical evidence (Schulz 1997), and the reporting methods are relatively consistent between trials, enabling a templated design. Also, the number of trials published by The BMJ is low (there were 7 in 2017), which allowed us to test out our ideas on a small number of articles.

Two randomised controlled trials were selected to receive the first visual abstracts, examining the effects of tai chi on fibromyalgia, and of emollient bath additives on eczema in children. Initial sketches were critiqued by research editors and other staff (see Figure 2).

After some refinement and experimentation, two final designs were produced (see Figure 3).

These abstracts gave an overview of the trials, including the characteristics of the population studied, the different health interventions compared, and the primary study outcomes. The abstracts also included a simple illustration to make them look visually distinct from one

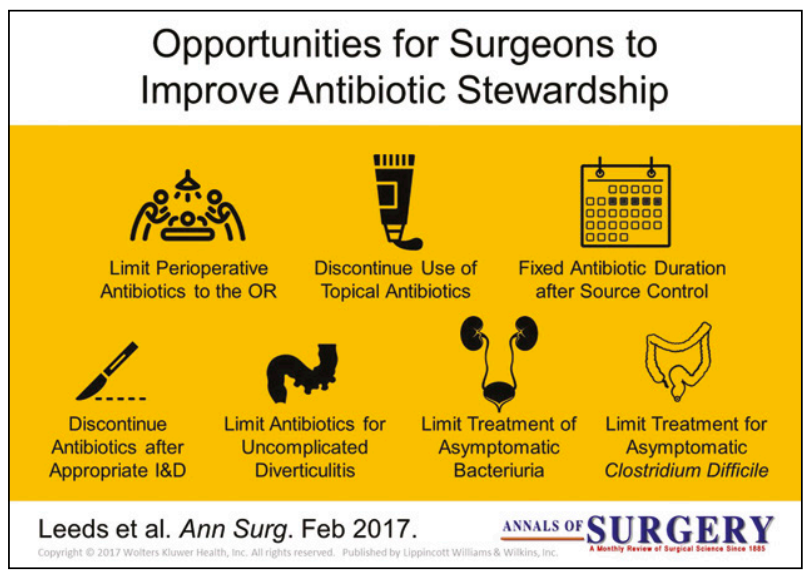

Figure 1. Examples of visual abstracts from Annals of Surgery (Ibrahim, A. M., 2016/17, reprinted with permission from the designer, copyright 2016/17). 


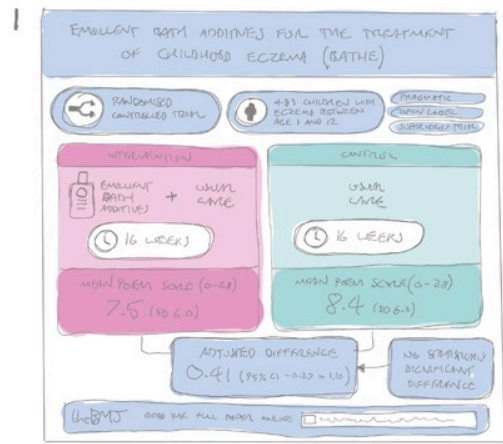

2
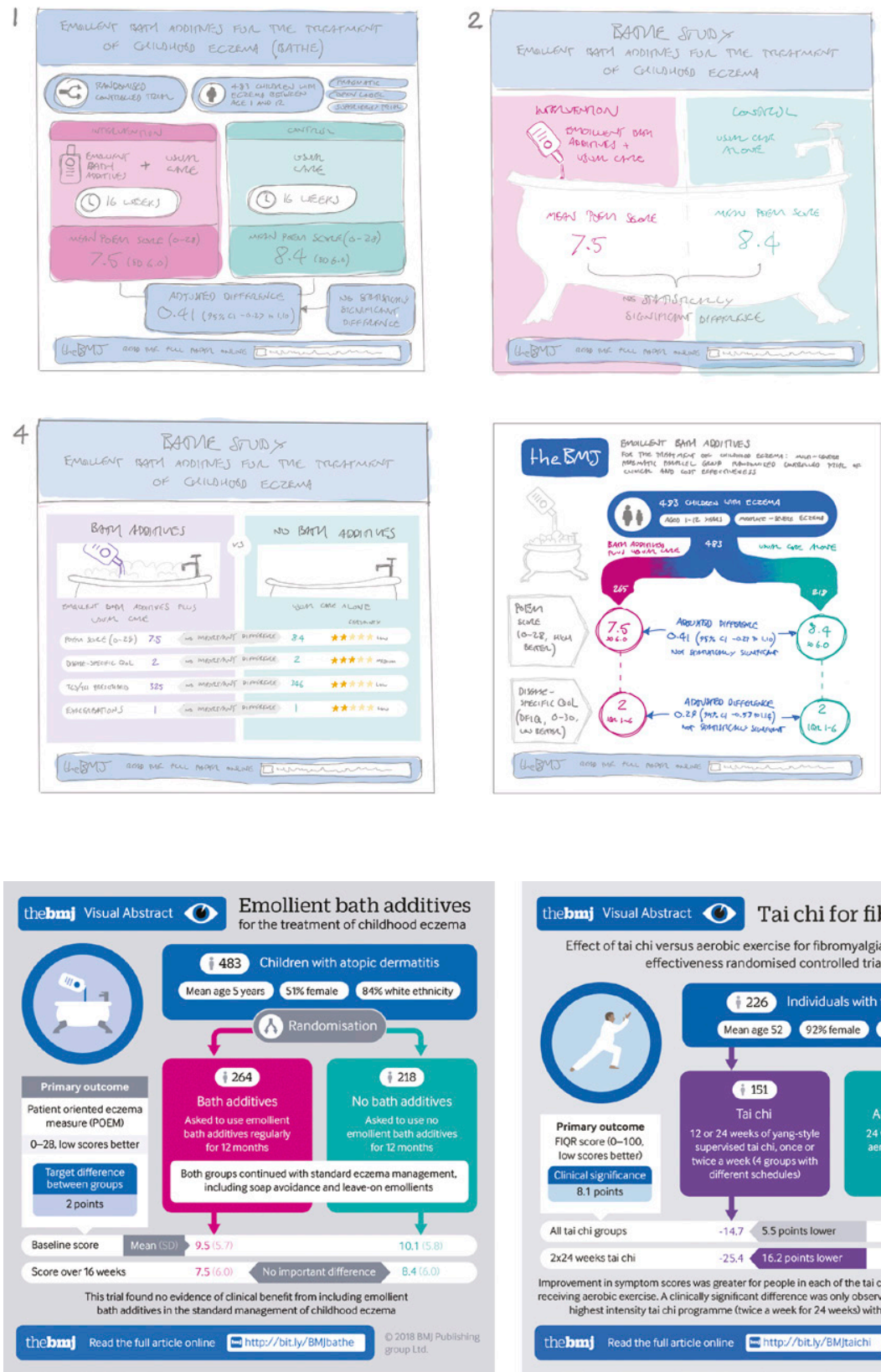

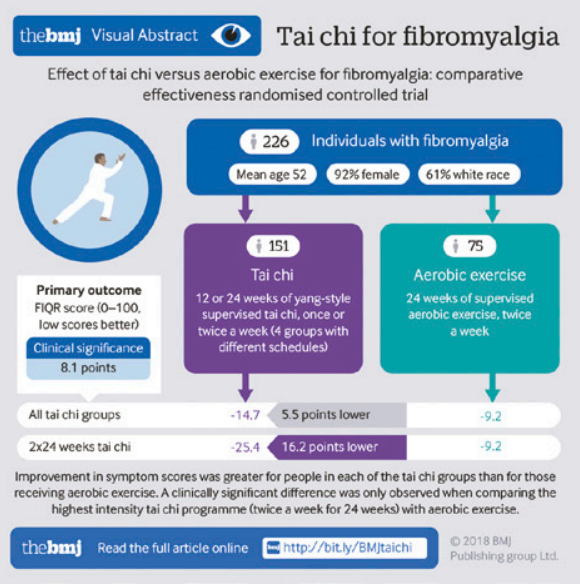

Figure 3. Final designs for two visual abstracts.
Figure 2. Initial designs for the bath additives visual abstract. 
another, and information about the clinical significance of the trial. At the bottom of the graphic, there is a short text description of the main finding, and a link to the article.

This information is broadly similar to the information provided in traditional abstracts, as set out in the CONSORT guidelines (Hopewell et al. 2008). However, to keep the abstracts to a $1024 \times 1024$ px size, not everything recommended could be included.

\section{Problem}

We report here on two evaluation methods that we used to assess readers' reactions to the new visual abstracts. This allowed us to highlight any problems with the new design that would need to be addressed.

\subsection{Social media performance}

The first visual abstract, on tai chi for fibromyalgia, was published on 21 March 2018 and by the end of 2018 we had published a total of 18 visual abstracts.
The social media statistics for the first visual abstract posted were encouraging (see Figure 4). Tweets including the new visual abstract received substantially more impressions, likes, and retweets than the average for all tweets from our main account, @bmj_latest, in March 2018. The tweets with the visual abstract also had more impressions, likes, and retweets than average for tweets containing other infographics that month. URL clicks were slightly lower than average for tweets containing other infographics, but higher than average for all @bmj_latest tweets.

However, there appears to have been a "halo effect" surrounding the first abstract, as Twitter performance over the course of the first year has not been as strong (see Figure 5). Generally, tweets about visual abstracts seem to receive a similar level of attention to the average of all our tweets, but slightly fewer URL clicks. They tend to receive less attention than our other graphics, which include more detailed full page visual summaries and interactive graphics.

\section{Mean Twitter stats, March 2018}

Visual abstract \#1 1 tweet

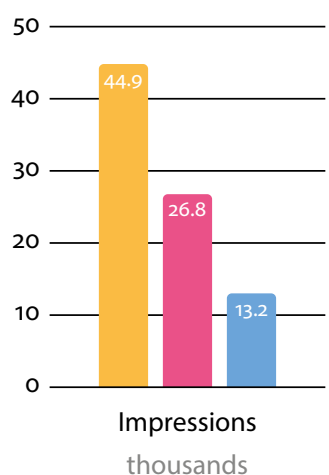

Other infographics 9 tweets

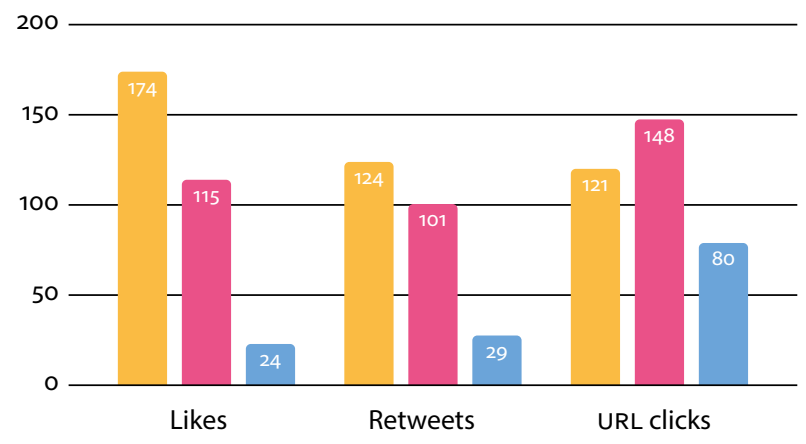

Figure 4. Stats from tweets about the first visual abstract (on tai chi for fibromyalgia). 


\section{Mean Twitter stats, June to December 2018}

Visual abstracts 32 tweets

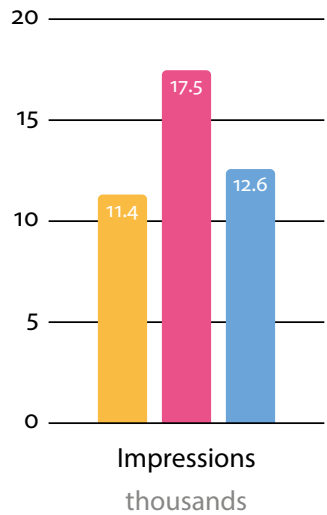

Other infographics 59 tweets

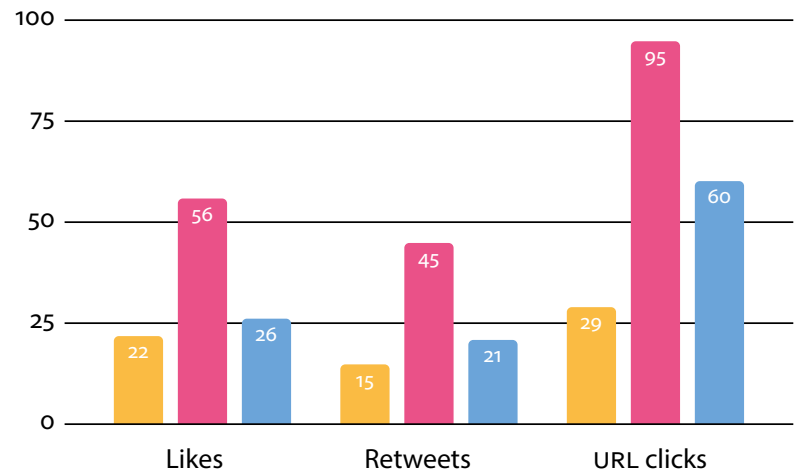

All tweets, @bmj_latest 3368 tweets
Figure 5. Performance of visual abstracts vs infographics and all tweets between June and December 2018.

\section{User survey}

While social media stats can show the popularity of visual abstracts, they do not explain why they are popular. To learn what readers thought about the design, and inform improvements, we designed a simple anonymised survey. This was adapted from pragmatic web usability methods that are widely used in digital publishing to assess user response to new features (Hall 2013; Krug 2010). Our survey was promoted via Twitter, and asked our readers to view our first visual abstract, then tell us whether they found it useful, why they found it useful or not useful, and how it could be improved. The survey also collected simple demographic data on the respondents' specialty, how often they treated patients with fibromyalgia, and the country in which they practised.

77 responses were recorded, 69 (89.6\%) of whom treated patients with fibromyalgia often or sometimes. 33 (42.8\%) respondents were general practitioners or primary care physicians. 55 of them answered the question about the usefulness of the visual abstract: $12(21.8 \%)$ found it "highly useful", 21 (38.1\%) found it "moderately useful", 16 (29.1\%) found it "a little useful" and $6(10.9 \%)$ found it "not useful at all".

We performed a sorting exercise on the qualitative responses, looking for themes (see Figure 6). Some participants who rated the visual abstract moderately or highly useful noted that they found the abstract easy to use, others noted that the treatment looked useful for their patients. Many of those who did not find the visual abstract as useful felt frustrated by the fact that tai chi was not available to their patients. A few people also mentioned specific data that could be included in the abstract, which was helpful for future development. Some participants wanted information that would be more suitable to give to patients, although this would likely require significantly more research and user testing than available to the team at present. 


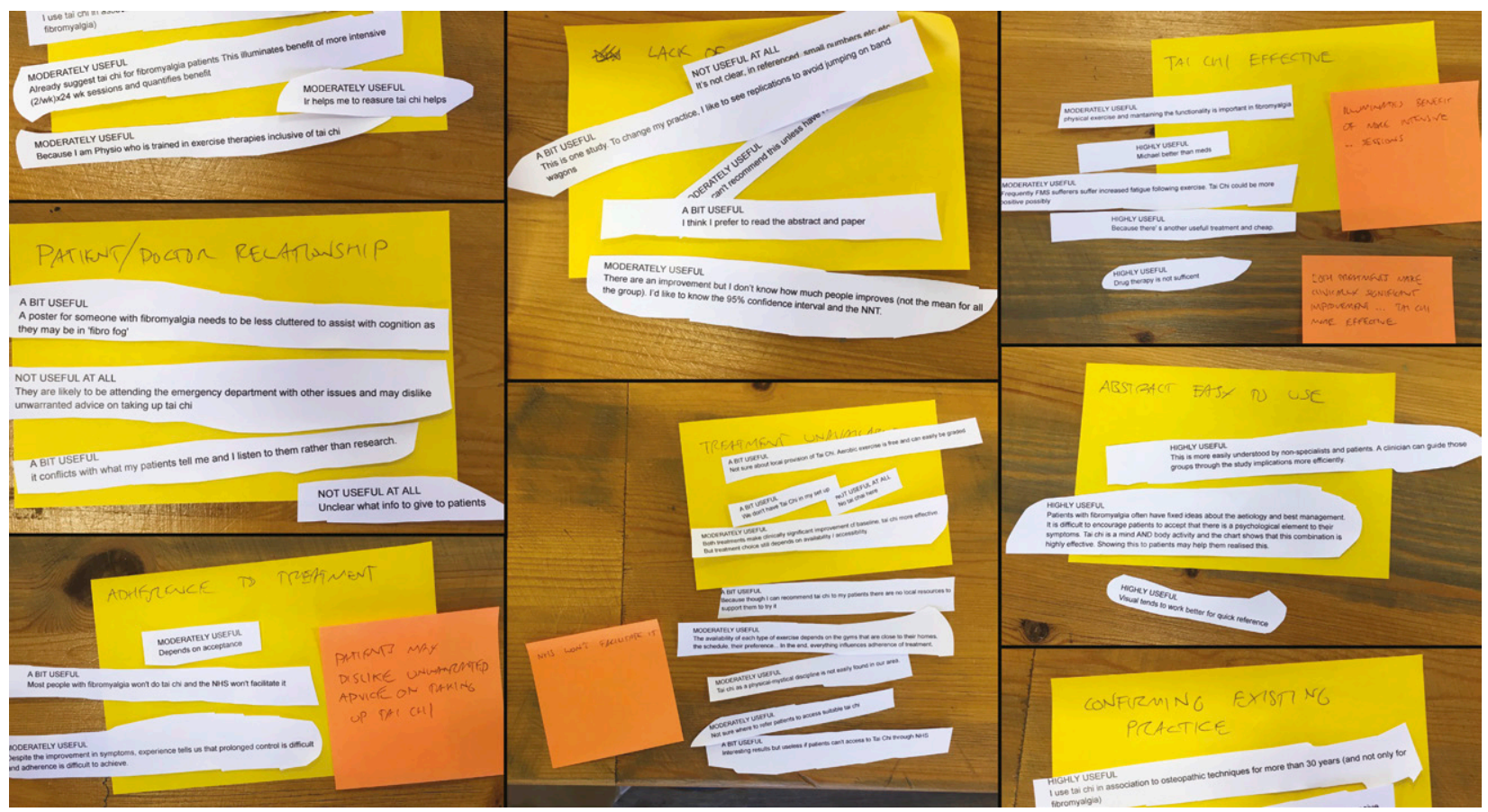

Figure 6. Sorted responses to the question "Please help us understand why you selected the answer above" in reference to how useful the participants found the information.

\section{Solutions}

Over the first year of creating visual abstracts, we have gradually incorporated several ideas from our initial studies. Given that the results were broadly favourable these changes have been minor.

One of the most important findings from our research was that participants reading the visual abstract were struggling to get an overview of the study findings until they had finished reading the entire abstract. To address this, we have moved the conclusion sentence to the top of the abstract. This convention is more typical of journalistic writing than scientific reporting, but it has been popular with our readers. We now also include a tick on a turquoise background for positive findings, or a cross on a pink background for negative findings (see Figure 7, comparison 1).

Another common theme from the survey was requests for more detailed statistics for doctors to judge how significant the study findings are. It is always challenging to get the level of detail right in this kind of situation. Too much detail could be overwhelming, whereas too little can be frustrating. It is also somewhat surprising given continuing controversy about the utility of reporting the relatively arbitrary measure of statistical significance (Amrhein, Greenland, \& McShane. 2019). 
March 2018

October 2018

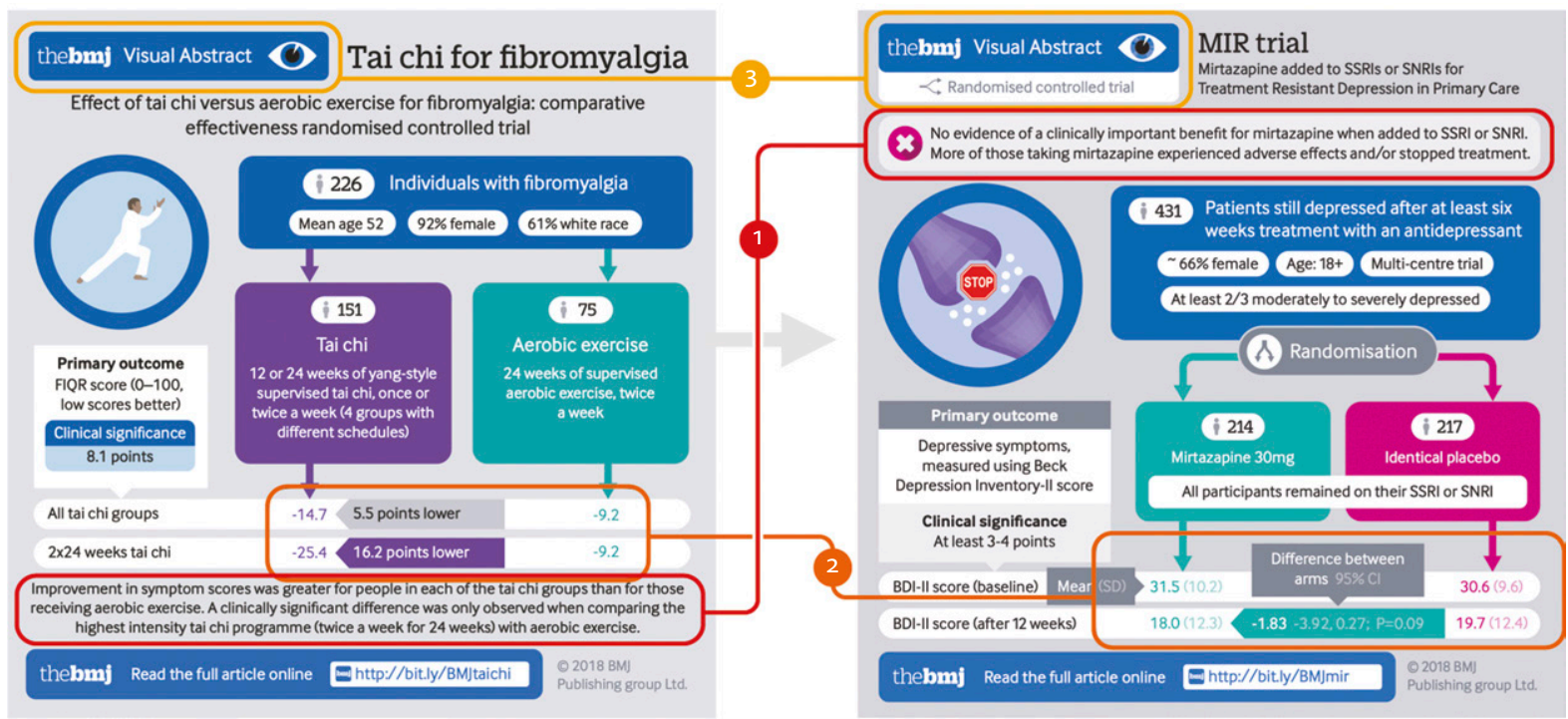

Figure 7. Changes suggested by survey results, incorporated into the visual abstract templates.

However, since readers find it a useful heuristic we have decided to include a measure of statistical significance (usually $95 \%$ confidence intervals or $P$-value) for the main findings (see Figure 7, comparison 2). We believe that this level of detail is more appropriate for the majority of our readers.

We have also added a white banner below the main "The BMJ visual abstract" branding to distinguish what kind of study is being reported (see Figure 7, comparison 3).

Some readers thought that the illustration was an unnecessary use of space, although this was not something we were prepared to remove. Illustrations serve an important role in attracting attention to something that may be relevant, whether on social media or on the printed page. However, this may not be a priority for participants presented with a single visual abstract to assess.

\section{Conclusions}

Our initial findings on social media performance and the results of the user study have been promising and The $B M J$ has now made visual abstracts a regular feature.

The results of our evaluation broadly align with existing research. The social media performance of the first abstract was impressive, replicating the findings of the Annals of Surgery (Ibrahim et al. 2017). However, caution should perhaps be taken when evaluating new formats using social media stats, as the visual abstracts published in the same format during the last 6 months of 2018 were less popular. This may be due to a "halo" effect of initial popularity around launching an innovative format.

In all our tests, URL clicks seemed lower than other measures such as impressions, likes and shares on Twitter. 
This may provide some clues about the findings in the Molecules journal study (Pferschy-Wenzig et al. 2016), which identified a lower rate of PDF downloads of papers with a visual abstract. It could be that our readers are more successfully triaging which papers to read, with fuller knowledge of the trial than would be provided by more traditional tweets.

Compared to social media stats, we found the user survey a more useful method of pragmatic design evaluation in a publishing business context. Questions that asked about the usefulness of the abstract and reasons why were most informative. These findings could then be grouped to draw out themes and make proposals for changes. This enabled rapid and inexpensive access to valuable feedback which helped us refine our template.

\section{Addendum: Post-Information+ redesign}

We would like to thank attendees of the Information+ conference in Potsdam, October 2018, for their feedback on the design of the template. Particular acknowledgement is due to Jaron Heard who suggested adding section headings and reducing the size of the link at the bottom of the abstracts. These comments have been included in a redesign of the original template, launched in January 2019 (see Figure 8).

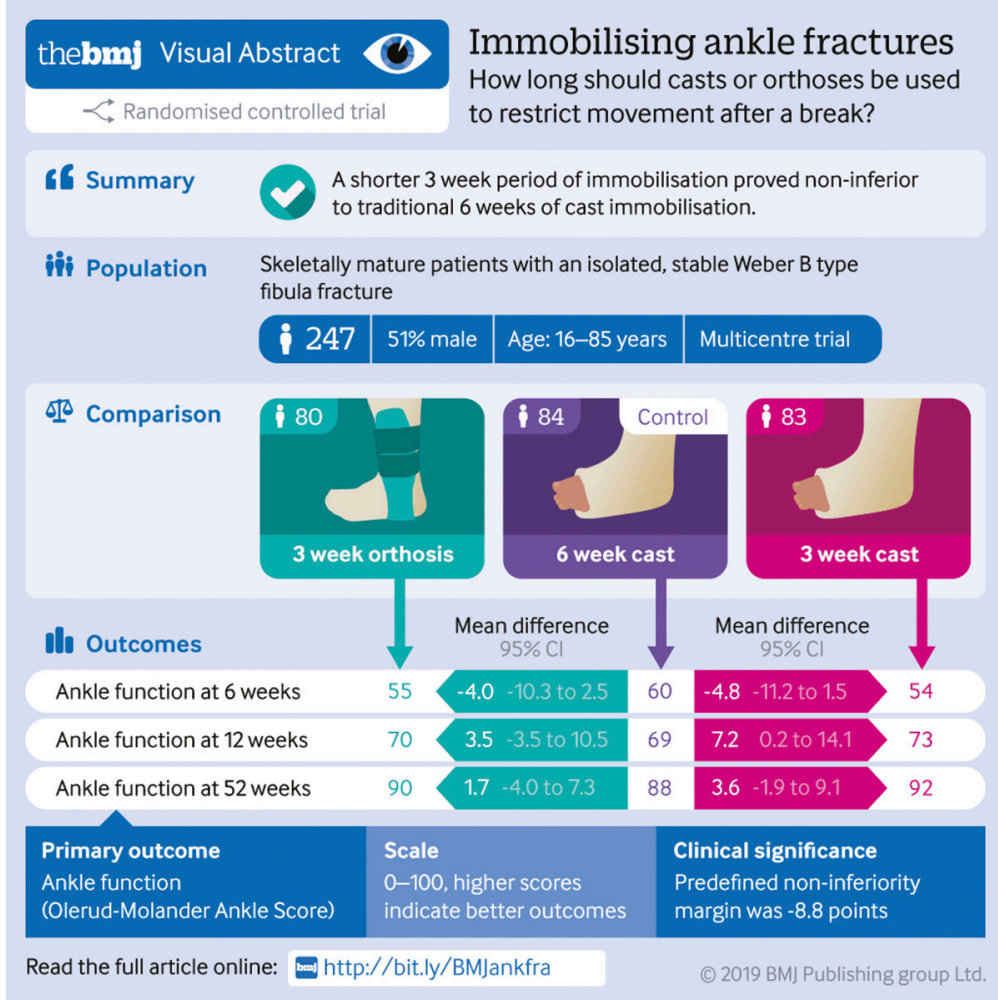

Figure 8. New template for the visual abstracts of The BMJ in 2019. 
Submission date: 1 February, 2019

Accepted date: 11 June, 2019

\section{References}

Amrhein, V., Greenland, S., \& McShane, B. (2019). Scientists rise up against statistical significance. Nature. Retrieved from https://www.nature.com/articles/d41586-019-00857-9

Cheng, K., Chen, Y., Larson, K., \& Rolandi, M. (2017). Proving the value of visual design in scientific communication. Information Design Journal, 23(1), 80-95. https://doi.org/10.1075/idj.23.1.o9che

Cooper, B. B. (2013). How Twitter's expanded images increase clicks, retweets and favorites. Retrieved from https://blog. bufferapp.com/the-power-of-twitters-new-expandedimages-and-how-to-make-the-most-of-it

Hall, E. (2013). Just enough research. New York, NY: A Book Apart. Hopewell, S., Clarke, M., Moher, D., Wager, E., Middleton, P., Altman, D. G., Schulz, K. F., the CONSORT Group (2008). CONSORT for reporting randomised trials in journal and conference abstracts. Lancet, 371(9609):281-283. Retrieved from http://www.equator-network.org/ reporting-guidelines/consort-abstracts/

Ibrahim, A. M., Lillemoe, K. D., Klingensmith, M., E., and Dimick, J. B., (2017). Visual abstracts to disseminate research on social media: A prospective, case-control crossover study. Annals of Surgery, 266(6), e46-e48.

https://doi.org/10.1097/SLA.0000000000002277

Krug, S. (2010). Rocket surgery made easy. Berkeley, CA: New Riders. Pferschy-Wenzig, E-M., Pferschy, U., Wang, D., Mocan, A., \&

Atanasov, A. G. (2016). Does a graphical abstract bring more visibility to your paper? Molecules, 21(9), 1247.

https://doi.org/10.3390/molecules21091247
Pinantoan, A. (2015). How to massively boost your blog traffic with these 5 awesome image stats. Retrieved from https:// buzzsumo.com/blog/how-to-massively-boost-your-blogtraffic-with-these-5-aw esome-image-stats/

Schulz, K. F. (1997). The quest for unbiased research: Randomized clinical trials and the consort reporting guidelines. Annals of Neurology, 41(5), 569-573.

https://doi.org/10.1002/ana.410410504

\section{About the author}

Will Stahl-Timmins is data graphics designer at The British Medical Journal. He creates infographics and data visualisations to support papers published at the journal, explaining complex research data and educational content for doctors in succinct

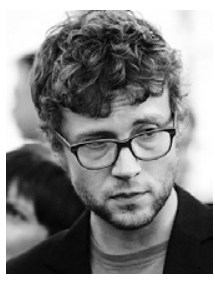
visual summaries. His background is in graphic design, and he holds a PhD in the visualisation of health research data from the University of Exeter Medical School in the UK.

Email:willstahl@gail.com

Jonathan Black is BMJ's user researcher. $\mathrm{He}$ uses qualitative research methods to help BMJ's product teams make decisions based on the needs of our users.

Email: jblack@bmj.com

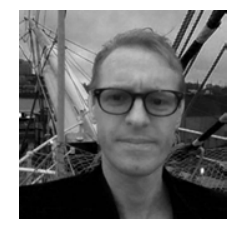

Paul Simpson is International Editor at The BMJ where he edits and manages the peer-review of scholarly articles.

Email:psimpson@bmj.com

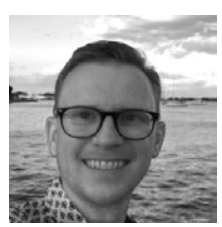

